Manuelle Medizin 2015 · 53:4-5 DOI 10.1007/s00337-015-1186-9

Online publiziert: 31. Januar 2015

(c) Springer-Verlag Berlin Heidelberg 2015

\author{
L. Beyer ${ }^{1} \cdot$ E.J. Seidel ${ }^{2}$ \\ 1 Ärztehaus Mitte, Jena \\ 2 Zentrum für Physikalische und Rehabilitative Medizin, Sophien- \\ und Hufeland-Klinikum Weimar gGmbH, Weimar
}

\title{
Manuelle Medizin und Musikermedizin
}

\section{Funktionale Medizin}

Liebe Leserin, lieber Leser,

im Namen des Herausgeberkollegiums von Manuelle Medizin wünschen wir Ihnen ein erfolgreiches und gesundes Jahr 2015.

Mit der Überarbeitung der Weiterbildungscurricula Manuelle Medizin (MM) und Manuelle Therapie sowie der Entwicklung von strukturierten modularen Weiterbildungskursen in osteopathischen Verfahren (Osteopathie) haben sich weitestgehend breit akzeptierte Techniken und Konzepte der manuellen Diagnostik und der manuellen Behandlung sowohl für Ärzte als auch für Physiotherapeuten herausgebildet. Dies wurde systematisch durch Beiträge der verschiedenen Schulen umfänglich dokumentiert. Ein weiterer Schritt war die Zusammenfassung von Befundkonstellationen zu regionalen Syndromen funktioneller Störungen und Schmerzen. Auch diese Beiträge wurden publiziert. Das Motto des gemeinsamen Kongresses 2014 der Deutschen Gesellschaft für Manuelle Medizin (DGMM) und der Deutschen Gesellschaft für Physikalische Medizin und Rehabilitation (DGPMR) „Funktionale Medizin“ weist auf den neu erreichten Stand in der MM hin, nämlich dass die vielfältigen Symptome der Hypomobilität, der muskulären Befunde, der Gewebeveränderungen und der sie begleitenden Schmerzen eigenständige Erscheinungen von Funktionsstörungen darstellen, die einer manuellen Therapie zugänglich sind.

Die Beiträge zur kraniomandibulären Dysfunktion (CMD) bzw. zum kra- niomandibulären System im vergangenen Heft verdeutlichen die fundamentalen Zusammenhänge (funktionalen Verkettungen) zwischen den verschiedenen Teilfunktionen der Aufrechterhaltung der Körperstatik bei allen Haltungen und Bewegungen. Die Erkenntnisse zur CMD und die Erfolge bei ihrer Behandlung haben dazu beigetragen, dass Manualmediziner und Kieferchirurgen bzw. Zahnärzte ein gemeinsames klinisches Substrat haben - die MM ist zum Partner der Zahnmedizin geworden, erste Ansätze standardisierter Therapien haben sich zu entwickeln begonnen. Eine ähnliche Entwicklung für multimodale Therapiekonzepte, die die MM einschließen, scheint es in der konservativen Orthopädie zu geben. Hier haben sich entsprechende Teams in den ANOA $^{1}$-Kliniken gebildet. Erste Ergebnisse aus der klinischen Forschung liegen vor.

\section{Musikermedizin als Modell zur Erklärung der Entstehung von Dysfunktionen}

Mit den im vorliegenden Heft dargestellten Ergebnissen zur Musikermedizin erschließt sich uns ein weiterer Bereich der funktionellen und manuellen Medizin, nämlich der Zusammenhang zwischen beruflicher Alltagsbelastung und der Entstehung von Dysfunktionen und Schmerzen in dessen Folge.

\footnotetext{
1 Arbeitsgemeinschaft nichtoperativer orthopädischer Akutkrankenhäuser
}

Die Beiträge zur Funktionsdiagnostik an der Wirbelsäule, zur statischen Belastung oder zur Belastung von Bindeund Stützgewebe beim Spiel der Querflöte (Günther et al.), beim Klavierspiel (Rau et al.), beim Gitarrenspiel (Seidel et al.) oder beim Spielen der Geige (Benz) können als einzigartige und typische Modelle der Entstehung von Funktionsstörungen bis zu Funktionserkrankungen gelten. Die betont einseitigen und überdurchschnittlich hohen spezifischen Belastungen bei den verschiedenen Berufsmusikern zeigen überdurchschnittlich häufig typische Entwicklungen von Dysfunktionen. Diese veränderten Funktionen sind größtenteils sogar die Voraussetzung für ein erfolgreiches Beherrschen des jeweiligen Instruments. Der gute Überblick über die funktionellen Sachverhalte am Bewegungssystem bei Musikern kann uns als Modell für die Erklärung der Entstehung von Funktionsstörungen dienen, das sich dann auf Ursache-Wirkungs-Beziehungen anderer Lebensbereiche übertragen lässt. $\mathrm{Zu}$ denken ist dabei an die Arbeitsmedizin mit den Bildschirmarbeitsplätzen, an Berufskraftfahrer und andere Beispiel oder auch an sportliche Freizeitbelastungen.

Wenn man die theoretischen Aspekte der Beiträge von Steinmetz sowie von Regenspurger $u$. Seidel in diesem Heft aufmerksam liest, lässt sich auch erkennen, dass die Dysfunktionen ein individuelles auf den Patienten ausgerichtetes therapeutisches Vorgehen erfordern und ermöglichen; das bedeutet, dass die MM ein hohes Potenzial zu einem individuellen Therapieansatz hat. 
Die dargelegten Beispiele aus der Musikermedizin sind so einleuchtend, dass sie als die notwendige Möglichkeit erscheinen, am Beispiel der Musiker die Ursache-Wirkungs-Kette für die Entstehung und Aufrechterhaltung von Dysfunktionen aus dem Theoriegebäude der MM den Ärzten in den verschiedenen Fachbereichen verständlich $\mathrm{zu}$ machen und sie so vom Konzept der MM/Osteopathie zu überzeugen. Gleiches gilt auch für die abgeleiteten Therapie- und Präventionsmaßnahmen, wenn diese auch noch unzureichend entwickelt sind.

\section{) Standardisierte}

\section{Therapie- und Präventions-}

\section{programme sind notwendig}

Steinmetz gibt zu diesen einzelnen Beispielen des Instrumentenspiels eine Literaturübersicht über die ,instrumentalspielassoziierten muskuloskeletalen Schmerzsyndrome (IAMS) bei professionellen Musikern". Sie verweist auch darauf, dass multimodale standardisierte Therapiekonzepte für die Behandlung von Musikern mit IAMS fehlen.

„Die hohen Prävalenzraten, die sich über fast 30 Jahre musikermedizinischer Forschung unverändert zeigen, fordern umfassende Therapiekonzepte und Präventionsmaßnahmen. Angesichts der hohen Chronifizierungsrate sowie zunehmender Hinweise auf psychosoziale Einflussfaktoren ist die Entwicklung multimodaler musikerspezifischer Therapieansätze, analog den etablierten Therapiekonzepten bei chronischen Schmerzen, notwendig."

Regenspurger $u$. Seidel führen hierzu aus: „Es gilt, extrinsische und intrinsische Risikofaktoren für die Entwicklung musikerspezifischer Beschwerden zu analysieren und darauf aufbauend ein multimodales Präventions- oder Behandlungsprogramm zu entwerfen. Geeignete Maßnahme hierfür sind manuelle Therapieverfahren, medizinische Trainingstherapie, Entspannungsverfahren, die Ökonomisierung von Spieltechnik und Übepraxis sowie eine verbesserte Ergonomie am Arbeitsplatz und am Instrument."

Hier liegen also die künftigen Aufgaben all derer, die sich mit manueller Dia- gnostik und manueller Behandlung intensiv beschäftigen, unabhängig davon, wie sie ihre empirischen Erkenntnisse bisher erworben haben, und unabhängig von den Schattierungen manualmedizinischer/osteopathischer Konzepte. Unter diesem Aspekt sollten Sie auch das von der DGMM herausgegebene Positionspapier zur „Osteopathie“ in Deutschland lesen.

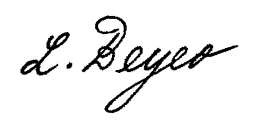

L. Beyer

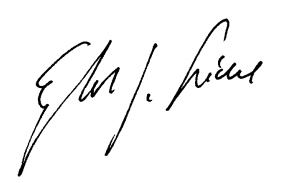

E. Seidel

\section{Korrespondenzadresse}

Prof. Dr. L. Beyer

Ärztehaus Mitte

Westbahnhofstr. 2, 07745 Jena

lobeyer@t-online.de

\section{Prof. Dr. E.J. Seidel}

Zentrum für Physikalische

und Rehabilitative Medizin, Sophien-

und Hufeland-Klinikum Weimar $\mathrm{gGmbH}$

Henry-van-de-Velde-Str. 2, 99425 Weimar

E.Seidel@Klinikum-Weimar.de

Interessenkonflikt. L. Beyer und E.J. Seidel geben an, dass kein Interessenkonflikt besteht.

\section{Medizinischer Nachwuchs profitiert von der Arbeit im Team.}

Medizinstudenten arbeiten in Zweierteams genauer als allein. Zu diesem Ergebnis kommt eine aktuelle Studie der Charité, die knapp 90 Medizinstudenten vor die Aufgabe stellte, am Computer simulierte Patientenfälle zu diagnostizieren. Alle angehenden Mediziner hatten einen vergleichbaren Wissensstand und sollten für jeweils sechs Patienten mit Atemnot diagnostische Tests anordnen. Anhand der erhaltenen Ergebnisse galt es, eine Diagnose auszuwählen - entweder als Einzelperson oder zu zweit. Im Team waren die Diagnosen akkurater und es entstehen $17 \%$ weniger Fehler. Meist gehen falsche Einschätzungen auf Denkfehler oder Fehler bei der Datenauswertung zurück. Zwar brauchen die Mediziner im Zweierteam etwas länger um zu ihrer Diagnose zu gelangen, die diagnostischen Tests, die sie anordnen, würden dagegen in einem realistischen klinischen Rahmen weniger Zeit beanspruchen. Berufsanfänger, die allein arbeiten, sichern sich stärker über aufwendige Diagnostika ab. Teams entscheiden ähnlich wie erfahrene Mediziner und arbeiten mit geringerem Diagnoseaufwand, folgern die Studienautoren. Dabei fühlen sich Paare sicherer hinsichtlich ihrer Diagnosestellung.

Literatur: Hautz WE et al (2015), JAMA doi:10.1001/jama.2014.15770.

Quelle: Charité Universitätsmedizin Berlin www.charite.de 